\title{
Cardiac tamponade as a cause of COVID-19
}

\author{
Oliver Robak ${ }^{1}$, Maciej Dudek², Jerzy R. Ladny ${ }^{2,3}$, Lukasz $\operatorname{Szarpak}^{2,4,5}$, \\ Natasza Gilis-Malinowska ${ }^{6}$, Michael Frass ${ }^{1}$ (i) \\ ${ }^{1}$ Department of Medicine I, Medical University of Vienna, Austria \\ ${ }^{2}$ Polish Society of Disaster Medicine, Warsaw, Poland \\ ${ }^{3}$ Chair of Emergency Medicine and Disaster, Medical University Bialystok, Poland \\ ${ }^{4}$ Maria Sklodowska-Curie Medical Academy in Warsaw, Poland \\ ${ }^{5}$ Maria Sklodowska-Curie Bialystok Oncology Center, Bialystok, Poland \\ ${ }^{6}$ First Department of Cardiology, Medical University of Gdansk, Poland
}

We read with great interest an article by Li et al. [1] which reviewed the cardiovascular complications in COVID-19 patients. The authors discuss many disease entities, including cardiac injury, myocarditis, acute coronary syndrome, heart failure, or sudden cardiac arrest. However, it is worth adding to this catalog cardiac tamponade, which is a pathological condition that directly threatens a patient's life. It consists in filling the pericardial cavity with a fluid that has the nature of an exudate or effusion, thus hindering filling the atria. Tamponade may arise because of infarction, trauma, mediastinal tumors and many other clinical conditions $[2,3]$.

Since December 2019, the world has been struggling with the pandemic of infections of severe acute respiratory syndrome coronavirus 2 (SARS-CoV-2), causing coronavirus disease 2019 (COVID-19). There is an increased recognition of cardiac involvement in patients with COVID-19 as it confers a worse prognosis. The most common cardiac complications include acute myocardial injury, arrhythmias, acute myocarditis and severe left ventricular dysfunction. However, several authors including Asif et al. [4] present a case of COVID-19-associated acute viral pericarditis complicated by large pericardial effusion and cardiac tamponade. Also, Hakmi et al. [5] showed that cardiac tamponade with concomitant biventricular failure can develop in COVID-19 patients.
As mentioned previously, cardiac tamponade is a life-threatening condition and, in the event of intensification of symptoms, requires urgent intervention in the form of decompression of the pericardial sac. However, in order not to delay the tamponade diagnosis procedure, especially in patients with suspected/confirmed COVID-19, when medical staff wears full personal protective equipment for aerosol generating procedures (PPE AGP), ultrasonography may be helpful $[6,7]$. For this purpose, the Focus Assessment Transthoracic in Emergency (FATE) protocol can be used.

The aim of the aforementioned is to perform basic echocardiographic projections - sub-sternal, apical, parasternal and bilateral pleural projections (in the middle axillary lines in the costal arch). The ultrasonographic images acquired in this way allows confirmation or exclusion of cardiac tamponade, and, with its presence under ultrasound control, it is also possible to drain it.

In conclusion, cardiac tamponade, which can also be associated with COVID-19 infection, is a therapeutic challenge requiring immediate confirmation and implementation of invasive treatment.

\section{Acknowledgements}

Study supported by the ERC Research NET and Polish Society of Disaster Medicine.

Conflict of interest: None declared

Address for correspondence: Lukasz Szarpak, Assoc. Prof. PhD, MBA, Maria Sklodowska-Curie Medical Academy in Warsaw, ul. Solidarności 12, 03-411 Warszawa, Poland, tel: +48 500186225, e-mail: lukasz.szarpak@gmail.com 


\section{References}

1. Li G, Saguner AM, An J, et al. Cardiovascular disease during the COVID-19 pandemic: Think ahead, protect hearts, reduce mortality. Cardiol J. 2020; 27(5): 616-624, doi: 10.5603/ CJ.a2020.0101, indexed in Pubmed: 32789839.

2. Sabatel-Perez F, Sastre-Perona MA, Alonso MB, et al. Extrapericardial cardiac tamponade due to massive retrosternal hematoma. Cardiol J. 2019; 26(5): 616-617, doi: 10.5603/CJ.2019.0105, indexed in Pubmed: 31701516.

3. Shibutani H, Yutaka K, Mukai Yu, et al. Cardiac tamponade secondary to right ventricular perforation caused by a temporary pacemaker lead in the course of myocardial infarction. Cardiol J. 2018; 25(4): 538-539, doi: 10.5603/CJ.2018.0087, indexed in Pubmed: 30211934.
4. Asif T, Kassab K, Iskander F, et al. Acute pericarditis and cardiac tamponade in a patient with COVID-19: a therapeutic challenge. Eur J Case Rep Intern Med. 2020; 7(6): 001701, doi: 10.12890/2020_001701, indexed in Pubmed: 32523921.

5. Hakmi H, Sohail A, Brathwaite C, et al. Cardiac tamponade in COVID-19 patients: Management and outcomes. J Card Surg. 2020 [Epub ahead of print], doi: 10.1111/jocs.14925, indexed in Pubmed: 32790006.

6. Pérez-Casares A, Cesar S, Brunet-Garcia L, et al. Echocardiographic evaluation of pericardial effusion and cardiac tamponade. Front Pediatr. 2017; 5: 79, doi: 10.3389/fped.2017.00079, indexed in Pubmed: 28484689.

7. Dudek M, Szarpak L, Ruetzler K. Application of interventional ultrasound in emergency medicine conditions. Disaster Emerg Med J. 2018; 3(4): 137-147, doi: 10.5603/demj.2018.0029. 